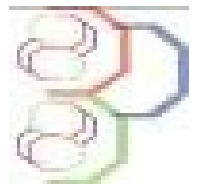

Journal of Applied Biosciences 134: 13702 - 13710

ISSN 1997-5902

\title{
Nouvelles variétés de canne à sucre (Saccharum officinarum L.) adaptées aux conditions pluviales dans les périmètres sucriers de Ferké au Nord Côte d'Ivoire
}

\author{
Crépin B. PÉNÉ, Mélanie B. BOUA², Yah COULIBALY-OUATTARA ${ }^{3}$,
}

1. Direction Recherche et Développement, SUCAF-Cl, bpene@sucafci.somdiaa.com

2. DRD/Service Études Agronomiques, SUCAF-Cl /Ferké 1, mboua@sucafci.somdiaa.com

3. DRD/Service Études Agronomiques, SUCAF-CI /Ferké 2, ycoulibaly@sucafci.somdiaa.com

Original submitted in on $8^{\text {th }}$ November 2018. Published online at www.m.elewa.org/journals/ on $28^{\text {th }}$ February 2019 https://dx.doi.org/10.4314/jab.v134i1.7

\section{RESUME}

Objectif : L'étude visait à améliorer la productivité agricole dans les plantations villageoises de canne à sucre par la sélection de variétés adaptées aux conditions pluviales.

Méthodes et Résultats : Chacun des trois essais agronomiques conduits en parcelles industrielles à Ferké 1 et Ferké 2 (Nord Côte d'Ivoire) a été disposé en blocs complets randomisés de Fisher avec une dizaine de variétés, dont un témoin, répétés 6 fois. Ces expérimentations ont été menées sur trois cycles consécutifs de récolte, soit une vierge et deux repousses, avec le rendement en canne et la tolérance aux maladies endémiques comme principaux critères de sélection. Les variétés M1176/77, M1246/84, M2593/92, M1565/87 et N21 se sont avérées significativement supérieures aux témoins R579 et SP701143. Quant aux variétés RB80/6043 et SP81/3250, elles sont apparues équivalentes au meilleur témoin R579.

Conclusion et application des résultats : Parmi ces 7 variétés sélectionnées en conditions pluviales, les plus productives devant faire l'objet de tests-précommerciaux étaient M1176/77 et N21 avec respectivement 82 et $85 \mathrm{t}$ de canne/ha au bout de 3 cycles de récoltes consécutifs depuis la nouvelle plantation. Pour impulser le développement de ces variétés tolérantes à la sécheresse dans les plantations industrielles ou villageoises conduites en conditions pluviales, elles sont multipliées chaque année dans les stations expérimentales en vue d'installer des pré-pépinières puis des pépinières destinées à produire des boutures nécessaires.

Mots-clés : rendement cane, tolérance à la sécheresse, tenue en repousse, récolte en vert, rang jumelé, sol ferralitique. 


\section{ABSTRACT \\ New Sugarcane (Saccharum officinarum L) Varieties Adapted to Rainfed Conditions in Ferké Sugar Plantations, Northern Ivory Coast}

Objective: The study aimed to improve agricultural productivity in village sugar cane plantations by selecting varieties adapted to rainfed conditions.Methods and results: Each of the three agronomic trials conducted in industrial plots at Ferké 1 and Ferké 2 (North Ivory Coast) was arranged in randomized complete blocks of Fisher with about ten varieties, including one control, repeated 6 times. These experiments were conducted on three consecutive cycles of harvest, one virgin and two regrowth, with cane yield and tolerance to endemic diseases as the main selection criteria. Performances of M1176/77, M1246/84, M2593/92, M1565/87 and N21 were significantly higher compared to the control varieties R579 and SP70/1143. As for RB80/6043 and SP81/3250, they were rather equivalent to the best control variety, namely R579.

Conclusion and application of results: Of these 7 varieties selected under rainfed conditions, the most productive pre-commercial tests were M1176 / 77 and N21 with respectively 82 and 85 t cane / ha after 3 consecutive harvest cycles since the plant cane. To enhance the development of these drought tolerant varieties on rainfed industrial or village plantation, they are grown on yearly basis at experimental stations to set up pre-nurseries then nurseries for the purpose of seed cane production.

Keywords: cane yield, drought tolerance, ratooning ability, green harvesting, double-row planting, ferralsol.

\section{INTRODUCTION}

La production sucrière en Côte d'Ivoire est essentiellement assurée à partir des plantations industrielles irriguées qui s'étendent sur environ 20000 ha pour l'ensemble des quatre complexes sucriers du pays (Péné et al, 1997). Toutefois, environ 1500 ha de plantations non irriguées ont été mises en place au début des années 1980 à la création du Complexe sucrier de Zuénoula, au Centre-Ouest du pays où la demande évaporative paraissait plus modérée par rapport à celle des périmètres sucriers installés au Nord (Ferké $1 \& 2$, Borotou-Koro). Avec la dégradation rapide ou l'obsolescence de certains systèmes d'irrigation équipant le parcellaire (aspersion classique par couverture intégrale ou par couverture partielle, aspersion par canon-enrouleur, goutte à goutte), l'on a assisté à l'accroissement inconsidéré des surfaces non irriguées dans les périmètres de Ferké, avec près de 2500 ha en 2015-16 (Péné et al, 2013, Péné et Assa, 2005). Le programme de développement des plantations villageoises de canne à sucre, initié depuis 1988 au complexe sucrier de Borotou-Koro où les conditions pédoclimatiques étaient favorables, visait à contribuer à la réduction des coûts de production du sucre en Côte d'Ivoire tout en permettant aux populations riveraines de s'approprier une nouvelle culture de rente pour améliorer leurs revenus. Ce programme a été par la suite étendu aux trois autres périmètres sucriers ivoiriens dont ceux de Ferké à la fin des années 1990, avec la privatisation de l'ex-SODESUCRE (société d'État), (Péné et Déa, 2000, Péné et al, 2001). Les plantations villageoises ont connu une nouvelle impulsion avec le projet d'extension des surfaces en cours de financement par l'UE dans le cadre de la relance de la filière sucre en Côte d'Ivoire. Au niveau des périmètres de Ferké, cela permettrait de passer de 2500 ha en 2015 à presque 3500 ha de plantations villageoises en 2017 (Péné et al, 2014). Force est de constater, toutefois, la faiblesse des rendements agricoles réalisés en conditions pluviales dans les plantations industrielles mais aussi et surtout dans les plantations villageoises où l'itinéraire technique n'est souvent pas respectés par les exploitants (Péné et Koulibaly, 2011, Péné et al, 2012, Kouamé et al, 2010b). Cette étude vise à améliorer la productivité agricole en culture pluviale de canne à sucre par la sélection de variétés adaptées à ce type d'exploitation afin de le rendre viable économiquement. 


\section{MATERIEL ET METHODES}

Caractéristiques des sites d'expérimentation: Chacune des trois expérimentations a été conduite dans une parcelle commerciale non irriguée. Les parcelles concernées étaient B2-09 à Ferké 1, d'une part, puis V4-51 et V4-52 à Ferké 2, d'autre part. Le sol en place dans ces parcelles, de type ferrallitique remanié avec une texture sablo-argileuse de couleur ocre, est marqué par une induration latéritique à moyenne profondeur $(70-75 \mathrm{~cm})$. C'est la texture dominante dans les deux périmètres sucriers de Ferké. Sa réserve en eau utile est en moyenne d'environ 90 $\mathrm{mm}$, soit une réserve facilement utilisable de $60 \mathrm{~mm}$ (Péné et Koulibaly, 2011). Le sol est majoritairement pauvre en matière organique ( $1.5 \%$ en moyenne) avec un $\mathrm{pH}$ fortement ou faiblement acide (6.0 à 6.5) et une faible capacité d'échange cationique ( 8 méq/100 g). Le climat de la région de Ferké est de type tropical sec avec un régime pluviométrique uni-modal centré sur les mois d'août et de septembre (Fig. 1). La saison humide s'étend d'avril à octobre et la saison sèche de novembre à mars. Le cumul annuel de pluies est d'environ $1200 \mathrm{~mm}$ dont presque la moitié est enregistrée en août-septembre. Le déficit pluviométrique moyen que subit une culture cannière non-irriguée à Ferké approche 600-700 mm (Péné et al, 2010). La saison sèche est marquée par une période très favorable à la maturation de la canne à sucre, celle de l'harmattan qui s'étend de mi-novembre à fin janvier, avec des écarts thermiques journaliers au-delà de 20 ${ }^{\circ} \mathrm{C}$ et une humidité relative de l'air atteignant parfois 30 $35 \%$. Les problèmes phytosanitaires endémiques de la canne à sucre en Côte d'Ivoire ont trait, d'une part, aux maladies du charbon (Ustilago scitaminea) et de l'échaudure foliaire (Xanthomonas albilineans) et, d'autre part, aux foreurs de tiges Sesamia calamistis et Eldana saccharina W qui sévissent aux stades juvénile et adulte de la canne à sucre, respectivement (Kouamé et al, 2010a, Péné et Kéhé, 2005).

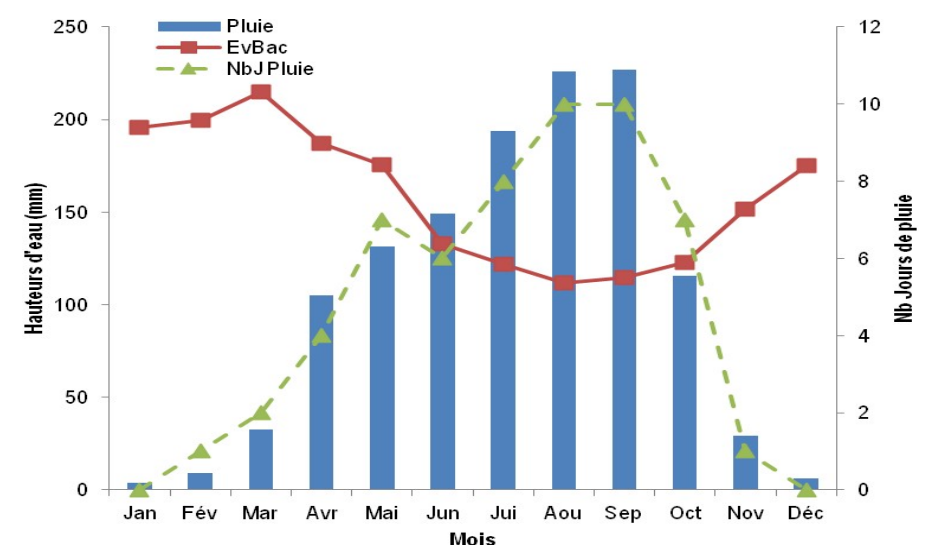

Figure 1 : Climat dans les périmètres sucriers de Ferké, Nord Côte d'Ivoire. Données moyennes décennales (20042017). [Climate in Ferké sugar mill plantations. Average decade data (2004-2017)]

Matériel végétal: Trois lots de matériel végétal comportant chacun une dizaine de variétés de canne à sucre d'origines diversifiées dont un témoin, ont été testées durant trois années consécutives, soit une nouvelle plantation, suivie de deux repousses. Deux variétés commerciales, en l'occurrence SP70/1143 et R579 très couramment exploitées à Ferké ont été utilisées comme témoins, respectivement, pour les essais de début et de fin de campagne agricole.

Dispositif expérimental: Chacune des trois expérimentations a été mise en place suivant un dispositif en blocs complets randomisés à un seul facteur étudié qui est la variété de canne à sucre, soit une dizaine par essai, avec 6 répétitions. Dans chaque essai, une micro-parcelle comportait 8 lignes de $10 \mathrm{~m}$ de long $\left(120 \mathrm{~m}^{2}\right)$, soit une surface totale de $7200 \mathrm{~m}^{2}$. Les deux principaux critères de sélection pris en compte pour la culture pluviale étaient le rendement en canne et la tolérance aux maladies et ennemis endémiques.

Conditions de culture : La plantation de la canne à sucre en simples rangs ( $1.5 \mathrm{~m}$ d'écartement) a été assurée manuellement en saison pluvieuse et cela manuellement, depuis la coupe des boutures jusqu'à leur mise en terre après les avoir préalablement tronçonnées. En dehors du matériel végétal utilisé, l'itinéraire technique pratiqué était le suivant : traitement herbicide au glyphosate de la culture cannière 

aux conditions pluviales dans les périmètres sucriers de Ferké au Nord Côte d'Ivoire

précédente, apports d'écume et de mélasse $(20$ t et 8 tha respectivement), décompactage du sol à $50 \mathrm{~cm}$ de profondeur dans sur les anciennes souches de canne plus riches en matières organique après ressuyage $d u$ sol consécutif à une bonne pluie $(20$ à $40 \mathrm{~mm}$ au moins), sillonnage, fertilisation N-P-K localisée assurée mécaniquement, plantation, traitement herbicide en pré-levée, sarclage à 4-5 et éventuellement 7-8 mois, récolte manuelle sur brûlis à 16 mois en canne vierge et à 11-12 mois. Les doses de fertilisation appliquées en culture pluviale en vierge ou en repousses étaient les suivantes: $100 \mathrm{~kg} \mathrm{~N} / \mathrm{ha}, 30 \mathrm{~kg} \mathrm{P} \mathrm{O}_{5} / \mathrm{ha}$ et $110 \mathrm{~kg}$ $\mathrm{K}_{2} \mathrm{O} / \mathrm{ha}$. Dans l'expérimentation conduite à Ferké 1 , les récoltes sur brûlis ont été effectuées successivement en vierge, 1 ère et 2è repousses à 16, 10.9 et 11 mois respectivement. Dans celle conduite à Ferké 2 sur la parcelle V4-52, les récoltes l'ont été à 16, 11.9 et 10.9 mois respectivement. Dans la 3è, conduite à Ferké 2 sur la parcelle V4-51, les récoltes l'ont été à 17, 10.9 et 12.7 mois, respectivement (Tab 1).

Tableau 1 Dates de plantation et de récolte selon les Lots de variétés testées en conditions pluviales et le périmètre sucrier à Ferké. [Planting and harvest dates following tested varieties under rainfed conditions and Ferké sugar mill plantation]

\begin{tabular}{l|c|c|c|c}
\hline Essais & Dates de & \multicolumn{3}{|c}{ Dates de récolte } \\
\hline variétaux & plantation & Vierge & Repousse 1 & Repousse 2 \\
\hline Lot 1 (Ferké 1) & $21 / 08 / 2010$ & $09 / 01 / 2012$ & $06 / 12 / 2012$ & $07 / 11 / 2013$ \\
\hline Lot 2 (Ferké 2) & $23 / 08 / 2010$ & $14 / 01 / 2012$ & $09 / 01 / 2013$ & $06 / 12 / 2013$ \\
\hline Lot 3 (Ferké 2) & $06 / 08 / 2011$ & $09 / 01 / 2013$ & $06 / 12 / 2013$ & $27 / 12 / 2014$ \\
\hline
\end{tabular}

Suivi agronomique en cours de végétation et à la récolte: Les observations phytosanitaires (comptage des fouets charbonneux/ha et des souches attaquées par l'échaudure foliaire) ont été réalisées à 4 mois et demi et les comptages de floraison effectués en novembre, soit à la fin de l'automne où l'intensité de la photopériode est maximale. A la récolte, après le comptage des tiges usinables dans les 2 lignes centrales de chaque micro-parcelle, 5 tiges usinables ont été prélevées au hasard dans celle-ci pour des mensurations agro-morphologiques (diamètre et

\section{RÉSULTATS}

Variations pluviométriques : À Ferké 1, 2011 et 2012 étaient les années déficitaires sur la période d'expérimentation par rapport à la moyenne pluviométrique décennale 2004-2014 et suivant l'indice de Nicholson (Paturel et al, 1997), contrairement à longueur de tiges, poids moyen d'une canne) suivies des analyses saccharimétriques effectuées séparément. Les cannes issues de 6 lignes centrales de chaque micro-parcelle, soit sur $90 \mathrm{~m}^{2}$ utiles, ont été pesées séparément pour déterminer le rendement en canne. Le comptage du nombre d'entrenœuds attaqués par le foreur de tiges Eldana Saccharina W a été effectué sur 50 tiges de canne prélevées au hasard dans l'andain correspondant à chaque micro-parcelle, parallèlement aux opérations de pesée.

2013 et 2014 qui étaient excédentaires (Tab 2). À Ferké 2, l'année 2011 qui était largement excédentaire avec un cumul de $1537 \mathrm{~mm}$ a été suivie d'une période déficitaire de 3 ans, soit de 2012 à 2014 .

Tableau 2 : Variations de la pluviométrie sur la période d'étude dans les périmètres sucriers de Ferké. [Rainfall variations over the period of study in both Ferké $1 \& 2$ sugar mill plantations]

\begin{tabular}{l|l|l|l|l|l}
\hline Périmètre & \multicolumn{4}{|c|}{ Cumuls de pluies sur période étudiée (mm) } & Pluie moyenne \\
\hline sucrier & 2011 & 2012 & 2013 & 2014 & Décenn. $(\mathrm{mm})$ \\
\hline Ferké 1 & 1183.5 & 1288.3 & 1242.1 & 1290.4 & $1228 \pm 141$ \\
\hline Ferké 2 & 1536.7 & 1318.1 & 1155.2 & 1158.3 & $1257 \pm 165$ \\
\hline
\end{tabular}

Indice de variabilité (Nicholson) : $(\mathrm{Xi}-\mu) / \sigma$; Xi : cumul de l'année i ; $\mu$ : pluie moyenne décennale ; $\sigma$ : écart-type

Variétés du lot 1 testées à Ferké 1

Caractéristiques biométriques: Les niveaux d'infestation constatés en 2è repousse sur le $1^{\text {er }}$ lot de variétés étaient faibles, soit 0 à 20 fouets charbonneux/ha et 0 à $2.5 \%$ d'entre-nœuds attaqués (ENA). Hormis les variétés M1334/84, VMC93/341 et 

aux conditions pluviales dans les périmètres sucriers de Ferké au Nord Côte d'Ivoire

VMC95/105, les autres ont présenté un fort taux de floraison en 2è repousse qui variait de 28 à $67 \%$ (Tab. 3). La longueur moyenne des tiges correspondant aux variétés SP80/1816 et M2593/92 étaient les plus élevées en 2è repousse avec 233 et $234 \mathrm{~cm}$ respectivement.

Tableau 3 : Moyennes des caractéristiques biométriques des variétés testées en cours de végétation et à la récolte (en 2è repousse) obtenues en conditions pluviales à Ferké 1. [Average biometrical traits of tested varieties during vegetation and at harvest (in $2^{\text {nd }}$ ratoon) obtained under rainfed conditions in Ferké 1

\begin{tabular}{|c|c|c|c|c|c|c|c|}
\hline \multirow{2}{*}{$\begin{array}{l}\text { Variétés } \\
\text { (lot 1) }\end{array}$} & \multicolumn{2}{|c|}{ En cours de végétation } & \multicolumn{5}{|c|}{$\dot{A}$ la récolte } \\
\hline & $\begin{array}{c}\% \\
\text { Floraison }\end{array}$ & $\begin{array}{l}\text { Nb fouets } \\
\text { charbon/ha }\end{array}$ & $\begin{array}{c}\mathrm{Nb} \text { tiges/ha } \\
\times 1000\end{array}$ & $\begin{array}{l}\text { Longueur } \\
\text { /tige (cm) }\end{array}$ & $\begin{array}{c}\text { Poids/canne } \\
(\mathbf{g})\end{array}$ & $\begin{array}{c}\text { Diamètrel } \\
\text { tige }(\mathrm{cm})\end{array}$ & $\%$ ENA \\
\hline M1176/77 & 50 & 12 & 79 & 198.5 & 991 & 26.7 & 0.7 \\
\hline M1400/86 & 46 & 1 & 77 & 215.5 & 918 & 21.5 & 2.2 \\
\hline M1334/84 & 8 & 0 & 77 & 196.9 & 1022 & 22.9 & 0.7 \\
\hline M1551/80 & 63 & 6 & 76 & 207.6 & 840 & 20.8 & 1.5 \\
\hline M2593/92 & 67 & 5 & 77 & 234.4 & 1008 & 21.0 & 1.2 \\
\hline SP79/2233 & 37 & 20 & 66 & 202.0 & 858 & 21.4 & 3 \\
\hline SP80/1816 & 28 & 17 & 60 & 233.3 & 1157 & 23.7 & 2.1 \\
\hline VMC93/341 & 12 & 2 & 69 & 205.2 & 840 & 22.7 & 1.6 \\
\hline VMC95/105 & 19 & 11 & 65 & 196.8 & 1090 & 23.7 & 1.8 \\
\hline SP70/1143 & 48 & 4 & 78 & 203.1 & 883 & 22.0 & 1.7 \\
\hline
\end{tabular}

Performances agronomiques à la récolte: Les variétés M1176/77 et $M$ 2593/92 se sont avérées significativement plus productives que le témoin avec, respectivement, 82 et 80 t de canne /ha soit des gains de 5 et $2 \%$ (Tab 4). Leurs rendements en sucre, d'une part, et teneurs en fibre, d'autre part, étaient acceptables avec environ 8 tha et $13 \%$ respectivement.

Tableau 4. Moyennes des qualités technologiques et des rendements agricoles des variétés testées en conditions pluviales à Ferké 1 (cumul V+2R). [Average yields and juice quality traits of tested varieties under rainfed conditions in Ferké 1 (All Plant cane and 2 ratoon crops)]

\begin{tabular}{|c|c|c|c|c|c|}
\hline Variétés & \multicolumn{3}{|c|}{ Qualités technologiques (\% Canne) } & \multicolumn{2}{|c|}{ Rendements } \\
\hline (lot 1) & Pureté & Pol & Fibre & Canne (t/ha) & Sucre (t/ha) \\
\hline M1176/77 & $86.9 \mathrm{~b}$ & $14.6 \mathrm{c}$ & $12.9 \mathrm{~b}$ & $81.9 \mathrm{e}$ & $8.7 \mathrm{~d}$ \\
\hline$\overline{\mathrm{M} 1400 / 86}$ & $84.5 \mathrm{a}$ & $12.4 \mathrm{a}$ & $12.3 \mathrm{a}$ & $73.3 \mathrm{c}$ & $6.4 \mathrm{a}$ \\
\hline M1334/84 & $86.2 \mathrm{~b}$ & $13.7 \mathrm{~b}$ & $13.0 \mathrm{~b}$ & $76.8 \mathrm{~d}$ & $7.5 \mathrm{~b}$ \\
\hline M1551/80 & $86.2 \mathrm{~b}$ & $13.6 \mathrm{~b}$ & $12.8 \mathrm{~b}$ & $68.3 \mathrm{~b}$ & $6.7 \mathrm{a}$ \\
\hline M2593/92 & $87.1 \mathrm{~b}$ & $13.5 \mathrm{~b}$ & $13.1 \mathrm{~b}$ & $79.7 \mathrm{e}$ & $7.8 \mathrm{bc}$ \\
\hline SP79/2233 & $90.1 \mathrm{~d}$ & $15.6 \mathrm{~d}$ & $13.4 \mathrm{~b}$ & $71.0 \mathrm{~b}$ & $8.3 \mathrm{c}$ \\
\hline SP80/1816 & $89.7 \mathrm{~cd}$ & $15.2 \mathrm{~d}$ & $14.1 \mathrm{c}$ & $66.5 \mathrm{ab}$ & $7.6 \mathrm{~b}$ \\
\hline VMC93/341 & $87.3 \mathrm{~b}$ & $14.2 \mathrm{c}$ & $14.9 \mathrm{~d}$ & $63.0 \mathrm{a}$ & $6.4 \mathrm{a}$ \\
\hline VMC95/105 & $88.9 \mathrm{~cd}$ & $15.1 \mathrm{~d}$ & $14.0 \mathrm{c}$ & $68.6 \mathrm{~b}$ & $7.7 \mathrm{bc}$ \\
\hline SP70/1143 (T) & $88.6 \mathrm{C}$ & $15.5 \mathrm{~d}$ & $15.0 \mathrm{~d}$ & $77.9 \mathrm{~d}$ & $8.8 \mathrm{~d}$ \\
\hline Moyenne & 87.6 & 14.3 & 13.5 & 72.7 & 7.6 \\
\hline CV $(\%)$ & 2.0 & 4.7 & 5.2 & 8.2 & 10.2 \\
\hline ETMoy & 1.7 & 0.7 & 0.7 & 6.0 & 0.8 \\
\hline Effet bloc & $* *$ & ** & ns & $* *$ & $* *$ \\
\hline Effet variétal & ** & ** & $* *$ & ** & ** \\
\hline Effet année & ** & ** & ** & ** & ** \\
\hline
\end{tabular}

Les moyennes suivies de la même lettre dans une colonne donnée ne sont pas significativement différentes au seuil de 5 ou $1 \%$ selon le test de DUNCAN. Ns: différences non significatives au seuil de $5 \%$., ${ }^{*}$ : différences significatives au seuil de $5 \%,{ }^{* *}$ : différences significatives au seuil de $1 \%$. 

aux conditions pluviales dans les périmètres sucriers de Ferké au Nord Côte d'Ivoire

Variétés du Lot 2 testées à Ferké 2

Caractéristiques biométriques: Les niveaux d'infestation de charbon constatés en 2è repousse sur le 2è lot de variétés testées étaient inférieurs ou égaux au seuil tolérable d'environ 5000 fouets/ha (Tab 5). Celles dues au foreur de tiges étaient inférieures au seuil de $5 \%$ d'entre-nœuds attaqués (ENA). Toutes les variétés de ce lot présentaient une floraison modérée en 2 è repousse, soit un taux variant de 12 à $24 \%$. En raison d'une pluviométrie faible et assez mal répartie en 2013 (1155 mm, contre 1320 en 2012), la longueur moyenne des tiges s'est avérée faible pour toutes les variétés, avec des valeurs allant de 105 à $134 \mathrm{~cm}$.

Tableau 5 : Moyennes des caractéristiques biométriques des variétés testées en cours de végétation et à la récolte (en 2è repousse) obtenues en conditions pluviales à Ferké 2. [Average biometrical traits of tested varieties during vegetation and at harvest (in $2^{\text {nd }}$ ratoon) obtained under rainfed conditions in Ferké 2]

\begin{tabular}{l|c|c|c|c|c|c}
\hline Variétés & \multicolumn{2}{|c|}{ En cours de végétation } & \multicolumn{4}{c}{ À la récolte } \\
\hline (lot 2) & $\begin{array}{c}\% \\
\text { Floraison }\end{array}$ & $\begin{array}{c}\text { Nb fouets } \\
\text { charbon/ha }\end{array}$ & $\begin{array}{c}\text { Nb tiges/ha } \mathbf{x} \\
\mathbf{1 0 0 0}\end{array}$ & $\begin{array}{c}\text { Longueur/ } \\
\text { tige } \mathbf{( c m})\end{array}$ & $\begin{array}{c}\text { Poids/canne } \\
\mathbf{( g )}\end{array}$ & $\%$ ENA \\
\hline $\mathrm{M} 1246 / 84$ & 18 & 4611 & 57 & 120 & 900 & 1.9 \\
\hline $\mathrm{M} 1400 / 86$ & 24 & 1778 & 60 & 113 & 520 & 1.8 \\
\hline $\mathrm{M} 1565 / 87$ & 18 & 3334 & 62 & 129 & 760 & 2.3 \\
\hline $\mathrm{VMC93/339}$ & 12 & 3389 & 49 & 120 & 920 & 2.0 \\
\hline $\mathrm{M} 1397 / 86$ & 18 & 5167 & 60 & 134 & 1080 & 2.1 \\
\hline $\mathrm{M} 1176 / 77$ & 22 & 5111 & 56 & 107 & 720 & 2.4 \\
\hline $\mathrm{M} 2593 / 92$ & 24 & 4111 & 64 & 129 & 740 & 2.2 \\
\hline $\mathrm{M} 2256 / 88$ & 23 & 1556 & 75 & 105 & 500 & 1.7 \\
\hline $\mathrm{M} 1042 / 86$ & 23 & 389 & 61 & 113 & 880 & 2.4 \\
\hline $\mathrm{SP70/1143(T)}$ & 18 & 722 & 63 & 108 & 820 & 2.3 \\
\hline
\end{tabular}

La variété M1176/77 est apparue moyennement sensible à l'échaudure foliaire. [M1176/77 was found moderately sensitive to leaf scald and smut]

Performances agronomiques à la récolte: Les variétés M1176/77, M1246/84 et M 1565/87 se sont avérées significativement plus productives que le témoin, avec environ $69 \mathrm{t}$ de canne /ha soit un gain de
$23 \%$ (Tab 6). Leurs rendements en sucre, d'une part, et teneurs en fibre, d'autre part, étaient acceptables avec environ 7 tha et $14-16 \% \mathrm{C}$ respectivement.

Tableau 6 : Moyennes des qualités technologiques et des rendements agricoles des variétés testées en conditions pluviales à Ferké 2 (cumul V+2R). [Average yields and juice quality traits of tested varieties under rainfed conditions in Ferké 2 (All Plant cane and 2 ratoon crops)]

\begin{tabular}{l|c|c|c|c|c}
\hline Variétés & \multicolumn{2}{|c|}{ Qualités technologiques (\% Canne) } & \multicolumn{2}{c}{ Rendements } \\
\hline (lot 2) & Pureté & Pol & Fibre & Canne (t/ha) & Sucre (t/ha) \\
\hline M1246/84 & $87.0 \mathrm{e}$ & $15.0 \mathrm{~d}$ & $16.1 \mathrm{~d}$ & $68.3 \mathrm{c}$ & $7.2 \mathrm{~d}$ \\
\hline $\mathrm{M} 1400 / 86$ & $84.8 \mathrm{bc}$ & $13.8 \mathrm{ab}$ & $15.1 \mathrm{bc}$ & $64.1 \mathrm{~b}$ & $6.1 \mathrm{a}$ \\
\hline M1565/87 & $85.1 \mathrm{c}$ & $14.2 \mathrm{bc}$ & $15.5 \mathrm{c}$ & $69.2 \mathrm{c}$ & $6.9 \mathrm{~b}$ \\
\hline VMC93/339 & $87.3 \mathrm{e}$ & $15.7 \mathrm{e}$ & $15.6 \mathrm{c}$ & $58.0 \mathrm{a}$ & $6.5 \mathrm{a}$ \\
\hline $\mathrm{M} 1397 / 86$ & $82.5 \mathrm{a}$ & $13.3 \mathrm{a}$ & $14.2 \mathrm{a}$ & $59.0 \mathrm{a}$ & $5.4 \mathrm{bc}$ \\
\hline $\mathrm{M} 1176 / 77$ & $84.0 \mathrm{~b}$ & $14.1 \mathrm{bc}$ & $14.2 \mathrm{a}$ & $69.0 \mathrm{c}$ & $6.8 \mathrm{c}$ \\
\hline $\mathrm{M} 2593 / 92$ & $84.2 \mathrm{~d}$ & $13.6 \mathrm{ab}$ & $15.0 \mathrm{~b}$ & $66.9 \mathrm{bc}$ & $6.2 \mathrm{~b}$ \\
\hline $\mathrm{M} 2256 / 88$ & $86.4 \mathrm{~cd}$ & $15.8 \mathrm{f}$ & $16.0 \mathrm{~cd}$ & $60.5 \mathrm{a}$ & $6.8 \mathrm{a}$ \\
\hline M1042/86 & $85.9 \mathrm{~cd}$ & $14.8 \mathrm{c}$ & $14.9 \mathrm{~b}$ & $58.6 \mathrm{a}$ & $6.1 \mathrm{bc}$ \\
\hline SP70/1143 (T) & $86.7 \mathrm{~d}$ & $15.4 \mathrm{~d}$ & $15.7 \mathrm{c}$ & $56.2 \mathrm{a}$ & $6.2 \mathrm{~d}$ \\
\hline Moyenne & 85.4 & 14.6 & 15.2 & 63.0 & 6.4 \\
\hline CV (\%) & 2.2 & 6.1 & 6.1 & 8.0 & 11.8 \\
\hline ETMoy & 1.9 & 0.9 & 0.9 & 5.0 & 0.8 \\
\hline
\end{tabular}


Péné et al., J. Appl. Biosci. 2019 Nouvelles variétés de canne à sucre (Saccharum officinarum L.) adaptées aux conditions pluviales dans les périmètres sucriers de Ferké au Nord Côte d'Ivoire

\begin{tabular}{|c|c|c|c|c|c|}
\hline Effet bloc & ** & * & ns & ** & ** \\
\hline Effet variétal & ** & ns & ** & ** & ** \\
\hline Effet année & ** & ** & ** & ** & ** \\
\hline
\end{tabular}

Variétés du Lot 3 testées à Ferké 2

Caractéristiques biométriques: Hormis les variétés RB73/454 et surtout SP83/2847 qui présentaient 6500 et 21700 fouets charbonneux/ha en 2è repousse, les niveaux d'infestations observés étaient en-dessous du seuil tolérable de 5000 fouets/ha (Tab 7). Les attaques du foreur tiges observées étaient en-deçà du seuil tolérable ( $5 \%$ d'ENA). Toutes les variétés de ce lot présentaient une floraison faible à très faible en 2è repousse, soit un taux variant de 0 à $15 \%$. En raison d'une pluviométrie faible et assez mal répartie en 2014 comme en 2013 (1160 mm, contre 1320 en 2012), la longueur moyenne des tiges s'est avérée faible pour toutes les variétés, excepté N21 avec $239 \mathrm{~cm}$. Cela suggère une bonne capacité d'adaptation de celle-ci en conditions pluviales.

Tableau 7 : Moyennes des caractéristiques biométriques des variétés testées en cours de végétation et à la récolte (en 2è repousse) obtenues en conditions pluviales à Ferké 2. [Average biometrical traits of tested varieties during vegetation and at harvest (in $2^{\text {nd }}$ ratoon) obtained under rainfed conditions in Ferké 2]

\begin{tabular}{l|c|c|c|c|c|c}
\hline Variétés & \multicolumn{2}{|c|}{ En cours de végétation } & \multicolumn{4}{c}{ À la récolte } \\
\hline (lot 3) & $\%$ Floraison & $\begin{array}{c}\text { Nb fouets } \\
\text { charbon/ha }\end{array}$ & $\begin{array}{c}\text { Nb tiges/ha } \mathbf{x} \\
1000\end{array}$ & $\begin{array}{c}\text { Longueur/ } \\
\text { tige (cm) }\end{array}$ & $\begin{array}{c}\text { Poids/canne } \\
\text { (g) }\end{array}$ & $\%$ ENA \\
\hline R581 & 0 & 833 & 63 & 193 & 950 & 3.7 \\
\hline SP80/3280 & 14 & 2917 & 70 & 208 & 995 & 4.1 \\
\hline RB80/6043 & 1 & 2667 & 66 & 185 & 1000 & 2.5 \\
\hline SP81/3250 & 0 & 333 & 82 & 193 & 915 & 2.7 \\
\hline SP83/2847 & 0 & 21751 & 56 & 199 & 1030 & 4.3 \\
\hline SP71/1081 & 15 & 83 & 82 & 188 & 865 & 4.4 \\
\hline RB73/454 & 0 & 6584 & 26 & 179 & 1175 & 4.5 \\
\hline N21 & 4 & 1667 & 74 & 239 & 1040 & 3.0 \\
\hline R579 & 0 & 0 & 59 & 188 & 1260 & 3.5 \\
\hline
\end{tabular}

Performances agronomiques à la récolte: Seule la variété N21 s'est avérée significativement plus productive que le témoin, avec environ $86 \mathrm{t}$ de canne /ha soit un gain de $15 \%$ (Tab 8). Deux variétés avérées équivalentes au témoin, RB80/6043 et SP81/3250, ont produit respectivement 72 et $73 \mathrm{t}$ de cannes/ha. Les rendements en sucre de ces variétés étaient équivalents à celui du témoin, soit 6 à 7 tha. Ces trois variétés prometteuses se sont avérées significativement plus riches en fibre que le témoin avec des teneurs égales à $15.8,14.5$ et $16 \% \mathrm{C}$, respectivement. Ces teneurs en fibre élevées expliquent en partie leur richesse saccharine plutôt moyenne (12 à $13.5 \% \mathrm{C})$.

Tableau 8 : Moyennes des qualités technologiques et des rendements agricoles des variétés testées en conditions pluviales à Ferké 2 (cumul V+2R). [Average yields and juice quality traits of tested varieties under rainfed conditions in Ferké 2 (All Plant cane and 2 ratoon crops)]

\begin{tabular}{l|l|l|l|l|l}
\hline Variétés & \multicolumn{4}{l|}{ Qualités technologiques (\% Canne) } & \multicolumn{2}{l}{ Rendements } \\
\hline (lot 3) & Pureté & Pol & Fibre & Canne (t/ha) & Sucre (t/ha) \\
\hline R581 & $81.1 \mathrm{ab}$ & $13.0 \mathrm{~b}$ & $16.3 \mathrm{c}$ & $56.2 \mathrm{~b}$ & $4.8 \mathrm{a}$ \\
\hline SP80/3280 & $82.9 \mathrm{bc}$ & $13.3 \mathrm{~b}$ & $14.2 \mathrm{ab}$ & $63.0 \mathrm{c}$ & $5.8 \mathrm{~b}$ \\
\hline RB80/6043 & $83.9 \mathrm{bc}$ & $13.5 \mathrm{~b}$ & $14.5 \mathrm{~b}$ & $71.6 \mathrm{e}$ & $6.7 \mathrm{bc}$ \\
\hline SP81/3250 & $81.3 \mathrm{ab}$ & $12.9 \mathrm{~b}$ & $16.1 \mathrm{c}$ & $73.2 \mathrm{e}$ & $6.2 \mathrm{bc}$ \\
\hline SP83/2847 & $84.9 \mathrm{bc}$ & $13.7 \mathrm{~b}$ & $14.6 \mathrm{~b}$ & $65.4 \mathrm{~d}$ & $6.2 \mathrm{bc}$ \\
\hline SP71/1081 & $85.4 \mathrm{c}$ & $14.9 \mathrm{c}$ & $16.0 \mathrm{c}$ & $58.9 \mathrm{bc}$ & $6.1 \mathrm{bc}$ \\
\hline
\end{tabular}


Péné et al., J. Appl. Biosci. 2019 Nouvelles variétés de canne à sucre (Saccharum officinarum L.) adaptées aux conditions pluviales dans les périmètres sucriers de Ferké au Nord Côte d'Ivoire

\begin{tabular}{|c|c|c|c|c|c|}
\hline RB73/454 & $84.8 \mathrm{bc}$ & $13.9 \mathrm{~b}$ & $14.8 \mathrm{~b}$ & $51.2 \mathrm{a}$ & $5.0 \mathrm{a}$ \\
\hline $\mathrm{N} 21$ & $78.4 \quad \mathrm{a}$ & $12.0 \mathrm{a}$ & $15.8 \mathrm{c}$ & $85.7 \mathrm{f}$ & $6.6 \mathrm{bc}$ \\
\hline$\overline{\mathrm{R} 579}(\mathrm{~T})$ & $83.1 \mathrm{bc}$ & $13.5 \mathrm{~b}$ & $13.5 \mathrm{a}$ & $75.0 \mathrm{e}$ & $7.0 \mathrm{c}$ \\
\hline Moyenne & 82.9 & 13.4 & 15.1 & 66.7 & 6.1 \\
\hline CV (\%) & 3.7 & 6.9 & 7.5 & 8.0 & 12.3 \\
\hline ETMoy & 3.1 & 0.9 & 1.1 & 5.3 & 0.7 \\
\hline Effet bloc & ns & ns & $\mathrm{ns}$ & ** & ns \\
\hline Effet variétal & $* *$ & $* *$ & $\star * *$ & ** & $\star *$ \\
\hline Effet année & ** & ** & ** & ** & ** \\
\hline
\end{tabular}

\section{DISCUSSION}

Variétés de canne sélectionnées: Cette étude a montré que les variétés M1176/77, M2593/92, M1246/84, M1565/87 et N21 étaient plus productives que les deux témoins utilisés (SP70-1143 et R579). Toutefois, les variétés RB80/6043 et SP81/3250 se sont avérées équivalentes au témoin le plus performant (R579) qui est assez bien cultivé en conditions pluviales aussi bien dans les plantations industrielles que villageoises (PÉNÉ et al, 2012). Parmi toutes ces variétés sélectionnées, les plus productives étaient M1176/77 et N21, avec respectivement 82 et $85 \mathrm{t}$ de cannes/ha comme rendements moyens réalisés sur 3 cycles consécutifs de récoltes (vierge et 2 repousses), à comparer avec 40 à 50 t/ha et 35 à 40 tha réalisés dans les conditions pluviales respectivement en plantations industrielles et en plantations villageoises (Péné et Koulibaly, 2011). Ces bonnes performances suggèrent une bonne tenue en repousse de ces variétés. Elles se caractérisent du reste par un faible indice de surface foliaire qui compte parmi les critères pertinents de tolérance variétale à la sécheresse (Inman-Bamber et al, 2012 ; Smit and Singels, 2006 ; Inman-Bamber, 2005, Ramburan et al, 2010). Ces résultats expérimentaux ont motivé le développement récent des variétés $\mathrm{M} 1176 / 77$ et $\mathrm{N} 21$ en plantations villageoises depuis les campagnes sucrières 2012-13 et 2014-15, respectivement. Leur développement progressif en plantations villageoises au cours des prochaines années devrait être assuré au détriment des surfaces cultivées avec R579 et NCo376. Les mesures préventives de sanitation des pépinières en vigueur par le traitement des boutures à l'eau chaude devraient maintenir, en deçà du seuil tolérable, les niveaux d'infestations de la variété M1176/77, avérée

\section{CONCLUSION ET APPLICATION DES RESULTATS}

II ressort de cette étude que sept variétés se sont avérés tolérantes à la sécheresse, parmi lesquelles deux, en l'occurrence MM76/77 et N21, étaient faiblement ou moyennement sensible au charbon et à l'échaudure foliaire en conditions pluviales.

Contrôle de l'enherbement: II s'avère que les meilleures variétés adaptées en conditions pluviales ont un faible indice foliaire, d'où la nécessité de les planter en rangs jumelés afin de favoriser la couverture rapide du sol et contribuer ainsi au contrôle de l'enherbement à moindre coût. Celui-ci est avec l'eau de pluie, la profondeur du sol et les fertilisants, les premiers facteurs limitants des rendements en culture pluviale de canne à sucre dans le contexte de Ferké, marqué par la pauvreté du sol en matière organique.

Mode de récolte: Les variétés M1176/77, N21 et NCo376, à la différence de R579, ne sont pas autoépaillantes et se prêtent d'autant moins à la récolte manuelle en vert. Celle-ci, contrairement à la récolte sur brûlis pratiquée couramment dans les plantations villageoises de canne à sucre, permet d'assurer un paillis du sol qui favorise la conservation de l'humidité et constitue une source importante de matière organique pour le sol, tout en limitant l'enherbement. La sélection de variétés auto-épaillantes et adaptées en conditions pluviales devraient favoriser l'adoption de cette pratique culturale par les exploitants de canne villageoise car elle contribue significativement à l'abaissement des coûts de production du sucre. Le projet de sélection variétale à partir de semences de canne à sucre fournies par eRcane (Institut Réunionnais de Recherche Développement), mis en œuvre à SUCAF-Côte d'Ivoire depuis novembre 2014, devrait permettre sur le long terme la mise au point de variétés véritablement adaptées aux conditions pluviales par un processus de sélection intervenant plus précocement (dès le stade de pré-sélection).

hautement productives avec en moyenne respectivement 82 et $85 \mathrm{t}$ de cannes/ha au bout de trois cycles de récoltes consécutifs depuis la nouvelle 
plantation. Pour accompagner le développement de ces variétés dans les conditions pluviales, tant en plantations industrielles que villageoises, elles sont multipliées chaque année en stations expérimentales

\section{RÉFÉRENCES}

Inman-bamber $\mathrm{Ng}$, 2005. Water relations in sugarcane and response to water deficits. Field Crops Res. 92 (14): 185-202

Inman-bamber Ng, Laksshman P and Park S, 2012. Sugarcane for water limited environments. Theoretical assessment of suitable traits. Field Crops Res. 134 (12): 95-104.

Kouamé DK, Péné CB et Zouzou M, 2010. Évaluation de la résistance variétale de la canne à sucre au foreur de tiges tropical africain (Eldana saccharina Walker) en Côte d'Ivoire. J. Appl. Biosci. 26 : 1614-16-22.

Kouamé KD, Péné CB, Zouzou M, 2010. Criblage de variétés commerciales de canne à sucre prometteuses dans le périmètre sucrier de Ferké 2 au Nord de la Côte d'lvoire: Optimisation de la durée de sélection. Sci. Nat. 7(1) : 97-106.

Paturel JE, Servat E, Kouamé B, Lubes-Niel H, Ouédraogo $M$ et Masson JM, 1997. Climatic variability in humid Africa along the Gulf of Guinea Part II: an integrated regional approach. J. of Hydrol. 191: 16-36.

Péné CB, Assa DA, 2005. Performances de trois variétés de canne à sucre soumises au rationnement hydrique en pré-maturation au nord de la Côte d'Ivoire. Agron. Afr. 17(1): 718.

Péné CB, Assa A. et Déa GB. 2001. Interactions eau d'irrigation-variétés de canne à sucre en conditions de rationnement hydrique. Cahiers Agric. 10(4) : 243-253.

Péné $C B$, Déa GB, 2001. Interactions eau d'irrigationvariétés de canne à sucre en conditions de rationnement hydrique au Nord de la Côte d'Ivoire. R. Agric. Sucr. Maurice 79(2):1-21.

Péné CB, Boua $M$, Pons $P, 2014$. Screening of early and late-season sugarcane varieties on sprinkler irrigated Ferralsols in northern Ivory Coast. Poster presentation at the $20^{\text {th }}$ World Congress of Soil Science (International Union for Soil Science - IUSS), Jeju, (South Korea), June 8-13, 2014. In: 20 th IUSS Jeju Congress Proceedings. Available on www.iuss.org en vue de mettre en place des pré-pépinières puis des pépinières aux fins de produire des boutures nécessaires.

Péné, CB, Boua M, Viremouneix T, 2012. R579 une variété de canne à sucre très prometteuse en développement sous irrigation et en pluvial à Ferké au Nord Côte d'Ivoire. In: Actes Congrès Sucrier 2012 ARTAS/AFCAS, Ile de La Réunion, 10-14 sept 2012. Disponible sur www.afcas.fr

Péné CB, Chopart JL, Assa AD, 1997. Gestion de l'irrigation à la parcelle en culture de canne à sucre (Saccharum officinarum $L$ ) sous climat tropical humide, à travers le cas des régions nord et centre de la Côte d'Ivoire. Sécheresse $8(2): 87-98$.

Péné $C B$, Kéhé $M, 2005$. Performance de trois variétés de canne à sucre soumises au rationnement hydrique en pré-maturation au Nord de la Côte d'Ivoire. Agron. Afr. 17 (1): 7-18.

Péné CB, Koulibaly GS, 2011. Sugarcane yield variations in northern and central Ivory Coast as influenced by soil water balance over two critical growth stages. J. Agric. Sci. Technol. 5, $N^{\circ} 2$ (33): 220-225.

Péné CB, Ndiaye $S$ et Konan NC, 2013. Sprinkler irrigation and soil tillage practices in sugarcane plantations as influenced by soil texture and water storage in northern Ivory Coast. J. of Life Sci., David Publishing Online ISSN: 19347405. Vol 7 (3): 293-301.

Péné CB, Ouattara HM \& Koulibaly GS, 2012. Late season sugarcane performance as affected by soil water regime at the yield formation stage on commercial farms in northern Ivory Coast. J. of Life Sci., David Publishing Online ISSN: 1934-7405. Vol 6 (6): 644-651.

Ramburan S, Paraskevopoulos A, Saville G, Jones M, 2010. A decision support system for sugarcane variety selection in South Africa based on genotype-by-environment analyses. Expl. Agric. 46(2): 243-257.

Smit MA and Singels A, 2006. The response of sugarcane canopy development to water stress. Field Crops Res. 98 (2-3): 91-97. 